\section{Asociación entre el polimorfismo rs9939609 del gen FTO y marcadores de adiposidad en población adulta chilena}

\author{
FANNY PETERMANN $N^{1, \mathrm{a}, \mathrm{f}, *}$, MARCELO VILLAGRÁN $2, \mathrm{~b}, \mathrm{~g}, *$, \\ CLAUDIA TRONCOSO ${ }^{3, a}$, LORENA MARDONES ${ }^{2, b, g}$, \\ ANA MARÍA LEIVA, ${ }^{4, c, f}$, MARÍA ADELA MARTÍNEZ ${ }^{5, \mathrm{~b}, \mathrm{f}}$, \\ ALEXGARRIDO-MÉNDEZ ${ }^{6, \mathrm{~d}, \mathrm{~g}}$,FELIPEPOBLETE-VALDERRAMA ${ }^{7, \mathrm{~d}, \mathrm{f}}$, \\ CARLOS SALAS-BRAVO ${ }^{8, \mathrm{~d}, \mathrm{f}}$, ROBINSON RAMÍREZ-VÉLEZ ${ }^{9, \mathrm{e}, \mathrm{g}}$, \\ NATALIA ULLOA ${ }^{10, b, f, g}$, FRANCISCO PÉREZ-BRAVO ${ }^{11, b, g}$, \\ CARLOS CELIS-MORALES ${ }^{1,12, \mathrm{~d}, \mathrm{~g}}$ (En representación \\ de todos los integrantes del grupo ELHOC, \\ Epidemiology of Lifestyle and Health Outcomes in Chile)
}

\section{Association between FTO (ns9939609) genotype and adiposity markers in Chilean adults}

Background: Numerous studies have identified the role of Fat-mass-associated-gene (FTO) in the development of obesity. Aim: To investigate the association of FTO gene with adiposity markers in Chilean adults. Material and Methods: 409 participants were included in this cross-sectional study. The association between FTO (rs9939609) genotype and adiposity markers was determined using linear regression analyses. Adiposity markers included were: body weight, body mass index, fat mass, waist circumference, hip circumference and waist/hip ratio. Results: A fully adjusted model showed a significant association between FTO genotype and body weight (2.16 kg per each extra copy of the risk allele [95\% confidence intervals (CI): 0.45 to 3.87], $p=0.014)$, body mass index (0.61 kg. $\mathrm{m}^{-2}$ [95\% CI: 0.12 to 1.20$\left.], p=0.050\right)$ and fat mass $(1.14 \%$ [95\% CI: 0.39 to 1.89 ], $p=0.010$ ). The greater magnitude of association was found between the FTO gene and fat mass when the outcomes were standardized to $z$-score. Conclusions: This study confirms an association between the FTO gene and adiposity markers in Chilean adults, which is independent of major confounding factors.

(Rev Med Chile 2018; 146: 717-726)

Key words: Adiposity; Alpha-Ketoglutarate-Dependent Dioxygenase FTO; Genotype; Obesity.
'BHF Glasgow Cardiovascular Research Centre, Institute of Cardiovascular and Medical Sciences, University of Glasgow, Glasgow, United Kingdom.

${ }^{2}$ Departamento de Ciencias Básicas. Universidad Católica de la Santísima Concepción.

Concepción, Chile.

${ }^{3}$ CIEDE-USCS y Departamento de Ciencias Clínicas y Preclínicas, Facultad de Medicina. Universidad Católica de la Santísima

Concepción. Concepción, Chile.

${ }^{4}$ Instituto de Anatomía, Histología y Patología, Facultad de Medicina, Universidad Austral de Chile. Valdivia, Chile.

Instituto de Farmacia, Facultad de Ciencias, Universidad Austral de Chile. Valdivia, Chile. ${ }^{6}$ Escuela de Educación Física, Universidad San Sebastián. Concepción, Chile.

${ }^{7}$ Escuela de Kinesiología, Facultad de Salud, Universidad Santo Tomás, Sede Valdivia, Chile. ${ }^{8}$ Departamento de Educación Física, Facultad de Educación, Universidad de Concepción. Concepción, Chile.

${ }^{9}$ Centro de Estudios en Medición de la Actividad Física (CEMA), Universidad del Rosario. Bogotá, Colombia.

${ }^{10}$ Departamento de Bioquímica Clínica e Inmunología, Facultad de Farmacia y Centro de Vida Saludable de la Universidad de Concepción. Concepción, Chile.

"Laboratorio de Nutrigenómica, Departamento de Nutrición, Escuela de Medicina, Universidad de Chile. Santiago, Chile.

${ }^{12}$ Centro de Investigación en Fisiología del Ejercicio (CIFE), Universidad Mayor. Santiago, Chile.

${ }^{\text {aNutricionista. }}$

bBioquímico.

cProfesora de Biología y Química.

dProfesor de Educación Física.

eFisioterapeuta.

${ }^{\mathrm{f}} \mathrm{MsC}$

${ }^{9} \mathrm{PhD}$.

*FP y MV contribuyeron de igual manera en este manuscrito y deben considerarse primer autor compartido.

Los autores declaran no tener conflictos de interés.

Recibido el 13 de noviembre de 2017 aceptado el 17 de abril de 2018.

\section{Correspondencia a:}

Dr. Carlos Celis-Morales

BHF Glasgow Cardiovascular Research Centre 126 University Avenue. Glasgow University Glasgow, United Kingdom. G12 8TA carlos.celis@glasgow.ac.uk
I a obesidad es una enfermedad crónica, recurrente y progresiva que se ha transformado rápidamente en una epidemia mundial ${ }^{1}$. Según datos de la Organización Mundial de la Salud (OMS), en el año 2014 más de 600 millones de adultos a nivel mundial presentaban esta enfermedad, estimándose que las cifras aumentarán para el año $2030^{2,3}$. En Chile, de acuerdo a datos de la Encuesta Nacional de Salud 2016-2017, 74,2\% de la población presenta sobrepeso u obesidad ${ }^{4}$, 
lo que posiciona a Chile como el país que lidera el ranking de obesidad en América Latina ${ }^{5}$ y como uno de los diez países que presenta mayor obesidad a nivel mundial ${ }^{3}$.

Entre los principales factores de riesgo modificables para el desarrollo de obesidad se encuentran la inactividad física y una elevada ingesta energética ${ }^{6-8}$. Por otro lado, entre los factores de riesgo no modificables se encuentra la constitución genética. Desde la secuenciación del genoma humano en el año $2000^{9}$ ha habido un importante incremento en la identificación de genes asociados a condiciones y enfermedades complejas, siendo la obesidad una de las más estudiadas ${ }^{10-12}$. Si bien, a la fecha, más de 97 variantes genéticas asociadas a la obesidad han sido descubiertas, el gen FTO (Fat-mass-associated-gene) es el gen que ha presentado la mayor magnitud de asociación con obesidad. Desde que fue descubierto en el año $2007^{13}$, el SNP (polimorfismo de nucleótido único) rs9939609 es el que ha sido mayormente estudiado por su reconocido efecto en el aumento del índice de masa corporal (IMC) y en el riesgo de obesidad ${ }^{12,14}$, confirmándose su asociación en distintas poblaciones de adultos y niños a nivel mundial ${ }^{11,12,15,16}$.

A pesar de la elevada prevalencia de obesidad en Chile y de que diversos estudios han identificado el rol del gen FTO en el desarrollo de ésta, hasta la fecha ningún trabajo ha examinado la asociación entre el gen FTO con distintos marcadores de adiposidad en población adulta chilena. En este contexto, el objetivo de este estudio fue investigar la asociación del gen FTO con los distintos marcadores de adiposidad en población adulta chilena.

\section{Materiales y Métodos}

Estudio descriptivo transversal que incluyó a 409 individuos pertenecientes al estudio GENADIO (Genes, Ambiente, Diabetes y Obesidad) y que contaban con información disponible en relación al gen FTO. EL proyecto GENADIO fue realizado en Chile entre los años 2009 y 2011 con el objetivo de evaluar la prevalencia de factores de riesgo de enfermedades cardiovasculares. La población estudiada estaba compuesta por 472 residentes de ascendencia mapuche y europea de las regiones del Biobío y Los Ríos (247 mapuche y 225 europea), sin historial médico de enfermedad metabólica o cardiovascular y que al momento de la evaluación no estuvieran bajo ninguna prescripción de medicamentos, tal como ha sido descrito anteriormente ${ }^{17}$. La muestra fue calculada en base a los datos otorgados por el CENSO año 2002, en el cual se identificó que la población indígena total chilena constituía $4,6 \%$ de la población. Se escogió como grupo de intervención a los nativos mapuches, ya que son el grupo étnico más grande del país. El estudio contó con la aprobación de los comités de ética de la Universidad de Chile, Universidad de Concepción y Universidad de Glasgow. Todos los participantes firmaron su consentimiento informado previo a la recolección de datos.

\section{Variables antropométricas: marcadores de adiposidad}

La evaluación antropométrica se realizó por personal previamente capacitado, utilizando protocolos estandarizados ${ }^{18}$. El peso corporal y la talla fueron determinados con una balanza electrónica (TANITA TBF 300A, USA) y tallímetro (SECA A800, USA) con una precisión de $100 \mathrm{~g}$ y $1 \mathrm{~mm}$, respectivamente. El perímetro de cintura (PC) fue medido con una cinta métrica no distendible (SECA Modelo 201, USA). El estado nutricional fue clasificado en base a los puntos de corte sugeridos por la OMS: bajo peso: $<18,5 \mathrm{~kg} / \mathrm{m}^{2}$; normo peso: $18,5-24,9 \mathrm{~kg} / \mathrm{m}^{2}$; sobrepeso: $25,0-29,9 \mathrm{~kg} / \mathrm{m}^{2}$ y obesidad: $\geq 30,0 \mathrm{~kg} / \mathrm{m}^{2}{ }^{19}$. Los valores utilizados para definir obesidad central fueron los siguientes: $\mathrm{PC} \geq 102 \mathrm{~cm}$ y $\geq 88 \mathrm{~cm}$ en hombres y mujeres, respectivamente ${ }^{19}$. El perímetro de cadera fue medido alrededor del punto más ancho alrededor de la región de la cadera, aproximadamente al nivel de la sínfisis púbica, con la misma cinta métrica y fue expresado en $\mathrm{cm}$. Para la medición de grasa abdominal se utilizó el índice de cintura/cadera, el cual fue obtenido tras la división entre estas dos variables. La composición corporal se determinó mediante la medición de cuatro pliegues cutáneos (bicipital, subescapular, suprailiaco y tricipital) a través de un caliper Harpenden de pliegues cutáneos (Cranlea \& Company, UK) ${ }^{17}$. Se aplicó la ecuación de Durnin y Womersley para estimar el porcentaje de masa grasa $\operatorname{corporal}^{20}$.

\section{Determinación de variantes alélicas del gen FTO (Fat Mass Obesity-associated gene)}

Para la genotipificación del SNP rs9939609 del gen FTO, se obtuvo ADN genómico de leucocitos 
periféricos mediante el kit QIAamp DNA blood midi kit (QIAGEN, Ltd. UK). La discriminación alélica se realizó mediante PCR de tiempo real en termociclador ABI 7900-HT. La detección del polimorfismo rs 9939609 se realizó por la metodología TaqMan ${ }^{\circledR}$ Pre-Designed SNP Genotyping Assay. La secuencia [VIC/FAM] utilizada fue 'GGTTCCTTGCGACTGCTGTGAATTT[A/T] GTGATGCACTTGGATAGTCTCTGTT'. Esta tecnología se eligió por ser altamente sensible y específica, además de obtener los resultados en cortos períodos de tiempo. El diseño del ensayo de TaqMan utilizado para detectar el SNP rs9939609 se encuentra disponible comercialmente en la compañía Applied Biosystem bajo el siguiente número de identificación: C3009062010 (Applied Biosystems, Warrington, UK). Todos los análisis de las muestras fueron realizados en duplicados, con $98 \%$ de éxito en la determinación del genotipo.

\section{Variables sociodemográficas y de estilo de vida}

Los datos sociodemográficos (edad, sexo, zona de residencia, nivel educacional, ingreso económico, etnia) y los datos asociados con estilos de vida, como el tabaquismo, fueron recolectados mediante encuestas validadas ${ }^{17}$. La ingesta dietaria fue evaluada a través del registro alimentario de bebidas y alimentos en los últimos siete días, los cuales fueron previamente pesados en una balanza de cocina modelo kd- $404^{17}$.

Para la medición de la capacidad cardiorrespiratoria se utilizó el Chester Step Test y el resultado de la prueba fue expresado en METs (Metabolic-energy-equivalents), siguiendo las recomendaciones descritas por Buckley et al. ${ }^{21}$. Los niveles de actividad física (AF) y el tiempo sedentario fueron estimados por acelerometría de movimiento (Actigraph GTM1, USA). La intensidad de la AF y el gasto energético se determinaron mediante el algoritmo de Freedson ${ }^{22}$.

\section{Análisis estadístico}

Los datos de caracterización de la población estudiada son presentados como promedio y desviación estándar (DE) para variables continuas, y como porcentaje para variables categóricas. Las diferencias entre genotipo fueron determinadas con análisis de regresión para variables continuas y con el test $\chi^{2}$ para variables categóricas.

Para investigar la asociación entre el gen FTO y los marcadores de adiposidad (peso corporal, IMC, PC, perímetro de cadera, índice cintura/ cadera y porcentaje de grasa corporal), se realizó análisis de regresión lineal. El genotipo del SNP rs9939609 del gen FTO fue codificado siguiendo un modelo genético aditivo $(0=\mathrm{TT}$ - homocigoto para el alelo protector; $1=\mathrm{AT}$ - heterocigoto para el alelo de riesgo; 2 = AA - homocigoto para el alelo de riesgo), $\mathrm{y}$, posteriormente, mediante análisis de regresión lineal se estimó el incremento en la variable de adiposidad por cada copia adicional de la variante de riesgo (alelo A). Estos resultados fueron presentados como promedio o coeficiente beta con su respectivo $95 \%$ intervalo de confianza (95\% IC).

Para determinar cuál de los marcadores de adiposidad presentaba mayor asociación con el gen FTO, todas las variables fueron estandarizadas a $z$-score. Estos resultados fueron presentados como coeficiente beta estandarizados (DE) con su respectivo 95\% IC por cada copia adicional del alelo de riesgo del gen FTO.

En este estudio, la interacción entre el gen FTO y sexo, así como también la interacción entre el gen y etnia, no evidenció interacciones significativas con ninguno de los marcadores estudiados (resultados no mostrados). Por ende, los análisis no fueron estratificados por etnia (mapuche-europea), solo ajustado por estas variables (sexo y etnia).

Todos los análisis fueron ajustados por variables de confusión mediante la utilización de cuatro modelos estadísticos. Modelo 0: sin ajustar; Modelo 1: ajustado por edad, sexo, etnia, nivel educacional, ingreso económico, nivel socioeconómico y zona de residencia (urbano/rural); Modelo 2: ajustado por el Modelo 1, pero también por AF, tiempo sedente y tabaquismo; Modelo 3: fue ajustado por el Modelo 2, pero también por consumo de energía, proteínas, grasas totales, carbohidratos complejos, carbohidratos simples y alcohol. La distribución del Hardy-Weinberg Equilibrium de los alelos del gen FTO fue estimada mediante el test $\chi^{2}$. Para todos los análisis se utilizó el programa STATA SE v14. El nivel de significación fue definido como $\mathrm{p}<0,05$.

\section{Resultados}

En forma similar a lo reportado previamente para otras poblaciones ${ }^{23}$, la frecuencia de los alelos 
Tabla 1. Características de la población según genotipo del gen FTO (rs9939609)

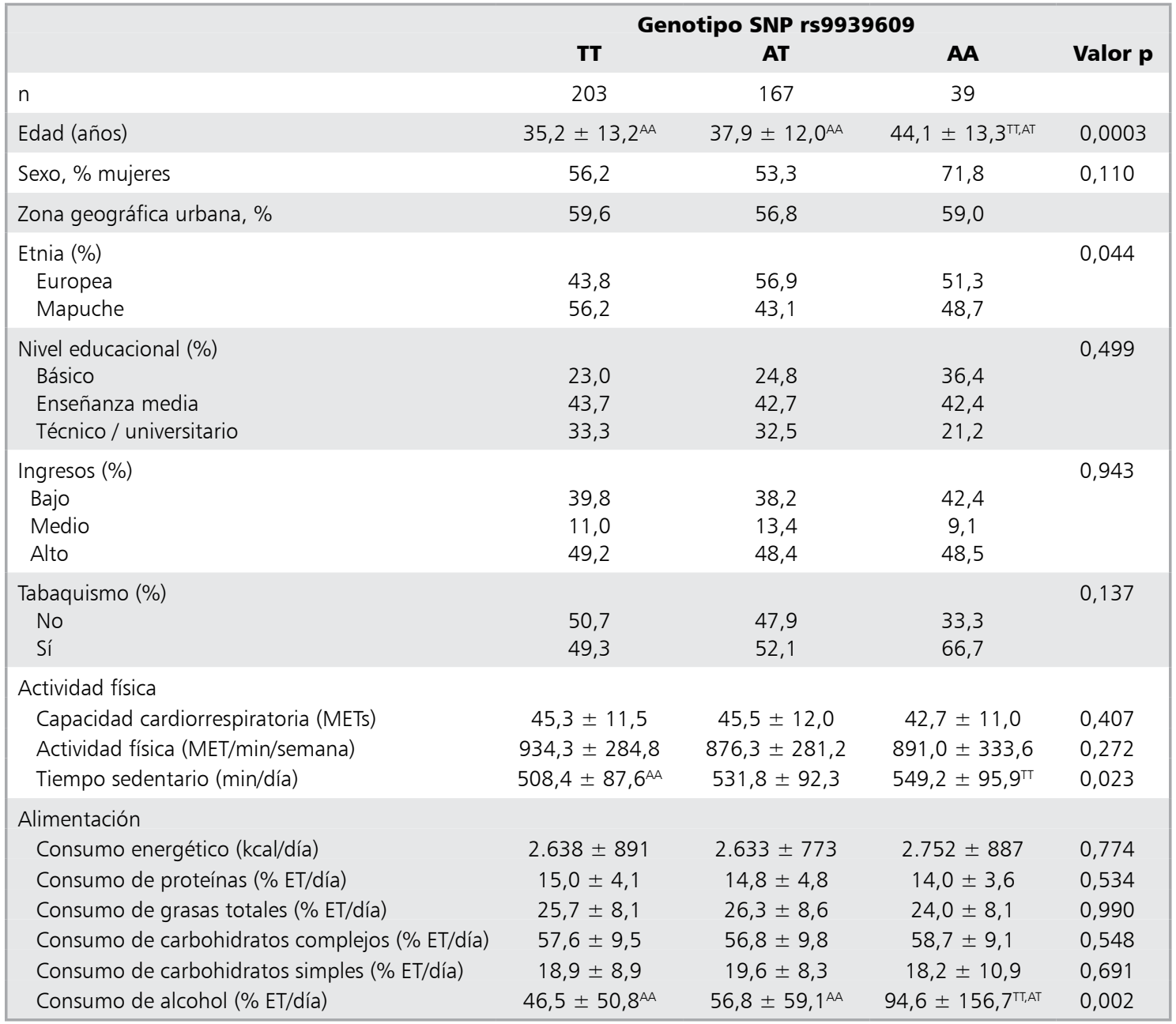

Datos presentados como promedio y desviación estándar para variables continuas y como \% para variables categóricas. Las diferencias entre genotipo fueron determinadas con análisis de regresión para variables continuas y con el test $\chi^{2}$ para variables categóricas. Se ha adicionado uno o más de un genotipo AA, AT, TT para diferenciar las variables que son estadísticamente significativas entre sí. ET: Energía total consumida.

del gen FTO se encuentran en Hardy-Weinberg Equilibrium (alelo $\mathrm{T}=0,701$ y alelo $\mathrm{A}=0,299$, $\left.\chi^{2}=0,296\right)$. En la Tabla 1 se presentan las características generales de la población según genotipo (AA, AT, TT). Los individuos con el genotipo AA predominante presentaban mayor edad y un mayor consumo de alcohol en comparación a individuos asociados a los genotipos AT y TT. No obstante, no se observaron diferencias significativas en los parámetros de AF, de alimentación ni en las variables sociodemográficas entre los indi- viduos con alelos de riesgo (AT y AA) respecto a individuos con el genotipo protector TT.

Los resultados de la asociación entre el gen FTO y variables de adiposidad son presentadas en la Tabla 2. Estos resultados revelan que, en el modelo no ajustado por variables de confusión, los marcadores de adiposidad incrementaron significativamente por cada copia extra del alelo de riesgo (A) del gen FTO ( $\mathrm{p}<0,0001)$. El incremento en los marcadores de adiposidad por cada copia del alelo de riesgo fue equivalente a $3,62 \mathrm{~kg}$ para peso 
Tabla 2. Asociación entre el gen FTO (rs9939609) y marcadores de adiposidad

\begin{tabular}{|c|c|c|c|c|c|}
\hline \multirow[b]{2}{*}{ Variables } & \multicolumn{3}{|c|}{ Genotipo del FTO (rs9939609) } & \multirow{2}{*}{$\begin{array}{l}\text { Efecto del modelo } \\
\text { genético aditivo }\end{array}$} & \multirow[t]{2}{*}{ Valor $\mathbf{p}$} \\
\hline & TT & AT & AA & & \\
\hline \multicolumn{6}{|c|}{ Peso corporal (kg) } \\
\hline Modelo 0 & $68,4(67,0 ; 69,9)$ & $71,9(70,3 ; 73,5)$ & $75,8(72,6 ; 79,1)$ & $3,62(2,08 ; 5,17)$ & $<0,0001$ \\
\hline Modelo 1 & $68,6(67,1 ; 70,1)$ & $71,1(69,6 ; 72,7)$ & $76,0(72,5 ; 79,5)$ & $3,24(1,61 ; 4,88)$ & $<0,0001$ \\
\hline Modelo 2 & $69,9(68,3 ; 71,4)$ & $71,1(69,4 ; 72,7)$ & $74,2(70,9 ; 77,5)$ & $1,93(0,28 ; 3,59)$ & 0,022 \\
\hline Modelo 3 & $69,4(67,9 ; 71,0)$ & $71,2(69,5 ; 72,9)$ & $74,0(70,6 ; 77,5)$ & $2,16(0,45 ; 3,87)$ & 0,014 \\
\hline \multicolumn{6}{|c|}{ IMC $\left(\mathrm{kg} / \mathrm{m}^{2}\right)$} \\
\hline Modelo 0 & $27,0(26,4 ; 27,5)$ & $28,5(28,0 ; 29,1)$ & $30,1(29,0 ; 31,3)$ & $1,58(1,03 ; 2,12)$ & $<0,0001$ \\
\hline Modelo 1 & $27,1(26,6 ; 27,7)$ & $28,4(27,8 ; 28,9)$ & $29,2(27,9 ; 30,5)$ & $1,10(0,51 ; 1,69)$ & $<0,0001$ \\
\hline Modelo 2 & $27,6(27,1 ; 28,1)$ & $28,3(27,7 ; 28,9)$ & $28,7(27,6 ; 29,9)$ & $0,61(0,03 ; 1,19)$ & 0,040 \\
\hline Modelo 3 & $27,5(27,0 ; 28,1)$ & $28,3(27,7 ; 28,9)$ & $28,6(27,4 ; 29,8)$ & $0,61(0,12 ; 1,20)$ & 0,046 \\
\hline \multicolumn{6}{|c|}{ Perímetro de cintura $(\mathrm{cm})$} \\
\hline Modelo 0 & $94,9(93,4 ; 96,3)$ & $97,3(95,7 ; 98,9)$ & $101,6(98,3 ; 104,9)$ & $3,06(1,49 ; 4,62)$ & $<0,0001$ \\
\hline Modelo 1 & $94,9(93,3 ; 96,4)$ & $97,5(95,8 ; 99,2)$ & $100,8(97,1 ; 104,5)$ & $2,86(1,15 ; 4,56)$ & 0,001 \\
\hline Modelo 2 & $95,7(94,0 ; 97,4)$ & $96,7(94,9 ; 98,5)$ & $100,1(96,5 ; 103,8)$ & $1,90(0,09 ; 3,72)$ & 0,040 \\
\hline Modelo 3 & $95,4(93,8 ; 97,0)$ & $96,0(94,2 ; 97,7)$ & $99,4(95,9 ; 102,9)$ & $1,66(-0,10 ; 3,42)$ & 0,065 \\
\hline \multicolumn{6}{|c|}{ Perímetro de cadera $(\mathrm{cm})$} \\
\hline Modelo 0 & $111,1(109,6 ; 112,5)$ & $113,6(111,9 ; 115,2)$ & $117,1(113,8 ; 120,5)$ & $2,86(1,27 ; 4,45)$ & $<0,0001$ \\
\hline Modelo 1 & $111,3(109,7 ; 112,9)$ & $113,8(112,1 ; 115,5)$ & $115,7(112,1 ; 119,4)$ & $2,32(0,60 ; 4,04)$ & 0,008 \\
\hline Modelo 2 & $111,6(109,8 ; 113,4)$ & $113,8(111,9 ; 115,4)$ & $114,5(110,7 ; 118,2)$ & $1,63(-0,26 ; 3,52)$ & 0,091 \\
\hline Modelo 3 & $111,3(109,6 ; 113,1)$ & $113,3(111,4 ; 115,2)$ & $113,4(109,5 ; 117,3)$ & $1,28(-0,65 ; 3,21)$ & 0,194 \\
\hline \multicolumn{6}{|c|}{ Índice de cintura/cadera } \\
\hline Modelo 0 & $0,94(0,93 ; 0,95)$ & $0,96(0,94 ; 0,97)$ & $0,95(0,92 ; 0,98)$ & $0,01(-0,01 ; 0,02)$ & 0,250 \\
\hline Modelo 1 & $0,94(0,93 ; 0,95)$ & $0,96(0,95 ; 0,97)$ & $0,95(0,92 ; 0,98)$ & $0,01(-0,00 ; 0,25)$ & 0,137 \\
\hline Modelo 2 & $0,94(0,92 ; 0,95)$ & $0,96(0,95 ; 0,98)$ & $0,95(0,92 ; 0,98)$ & $0,01(-0,00 ; 0,03)$ & 0,153 \\
\hline Modelo 3 & $0,94(0,92 ; 0,95)$ & $0,96(0,94 ; 0,97)$ & $0,95(0,91 ; 0,98)$ & $0,01(-0,01 ; 0,02)$ & 0,321 \\
\hline \multicolumn{6}{|c|}{$\%$ masa grasa } \\
\hline Modelo 0 & $29,0(28,4 ; 29,7)$ & $30,0(29,4 ; 30,7)$ & $31,8(30,5 ; 33,2)$ & $1,27(0,61 ; 1,93)$ & $<0,0001$ \\
\hline Modelo 1 & $28,8(28,1 ; 29,4)$ & $30,1(29,4 ; 30,8)$ & $31,7(30,2 ; 33,1)$ & $1,41(0,73 ; 2,09)$ & $<0,0001$ \\
\hline Modelo 2 & $28,5(27,9 ; 29,2)$ & $30,0(29,3: 30,7)$ & $30,6(29,3 ; 32,0)$ & $1,17(0,47 ; 1,86)$ & 0,001 \\
\hline Modelo 3 & $28,6(28,0 ; 29,3)$ & $30,2(29,4 ; 30,9)$ & $30,6(29,1 ; 32,1)$ & $1,14(0,39 ; 1,89)$ & 0,003 \\
\hline
\end{tabular}

Datos presentados como promedio y $95 \%$ IC según genotipo. El modelo genético aditivo indica el promedio de incremento en la variable de adiposidad por cada copia adicional de la variante de riesgo (A). Este efecto aditivo y su respectivo $95 \%$ IC fue determinado mediante regresión lineal. Los análisis fueron ajustados por: Modelo 0 - sin ajustar; Modelo 1 -ajustado por edad, sexo, etnia, nivel educacional, ingreso económico, nivel socioeconómico, y zona de residencia (urbano/rural); Modelo 2 - ajustado por el Modelo 1 pero también por actividad física, tiempo sedente y tabaquismo; Modelo 3 - fue ajustado por el Modelo 2 pero también por consumo de energía, proteínas, grasas totales, carbohidratos complejos, carbohidratos simples y alcohol.

corporal, $1,58 \mathrm{~kg} / \mathrm{m}^{2}$ para IMC, $3,06 \mathrm{~cm}$ para $\mathrm{PC}$, $2,86 \mathrm{~cm}$ para perímetro de cadera y en $1,27 \%$ para masa grasa. Al ajustar los modelos por el Modelo 2, la magnitud de las asociaciones se redujo, pero permanecieron significativas para peso corporal, IMC, PC y \% de masa grasa, mientras que no fue significativa para perímetro de cadera. Al ajustar los modelos por variables alimentarias (Modelo 3), la magnitud de la asociación por cada copia adicional del alelo de riesgo del gen FTO disminuyó a $2,16 \mathrm{~kg}$ para peso corporal, $0,61 \mathrm{~kg} / \mathrm{m}^{2}$ para IMC y $1,14 \%$ para masa grasa. Sin embargo, la asociación no fue significativa para PC (Tabla 2 y Figura 1 ). No se encontraron asociaciones entre el gen FTO 


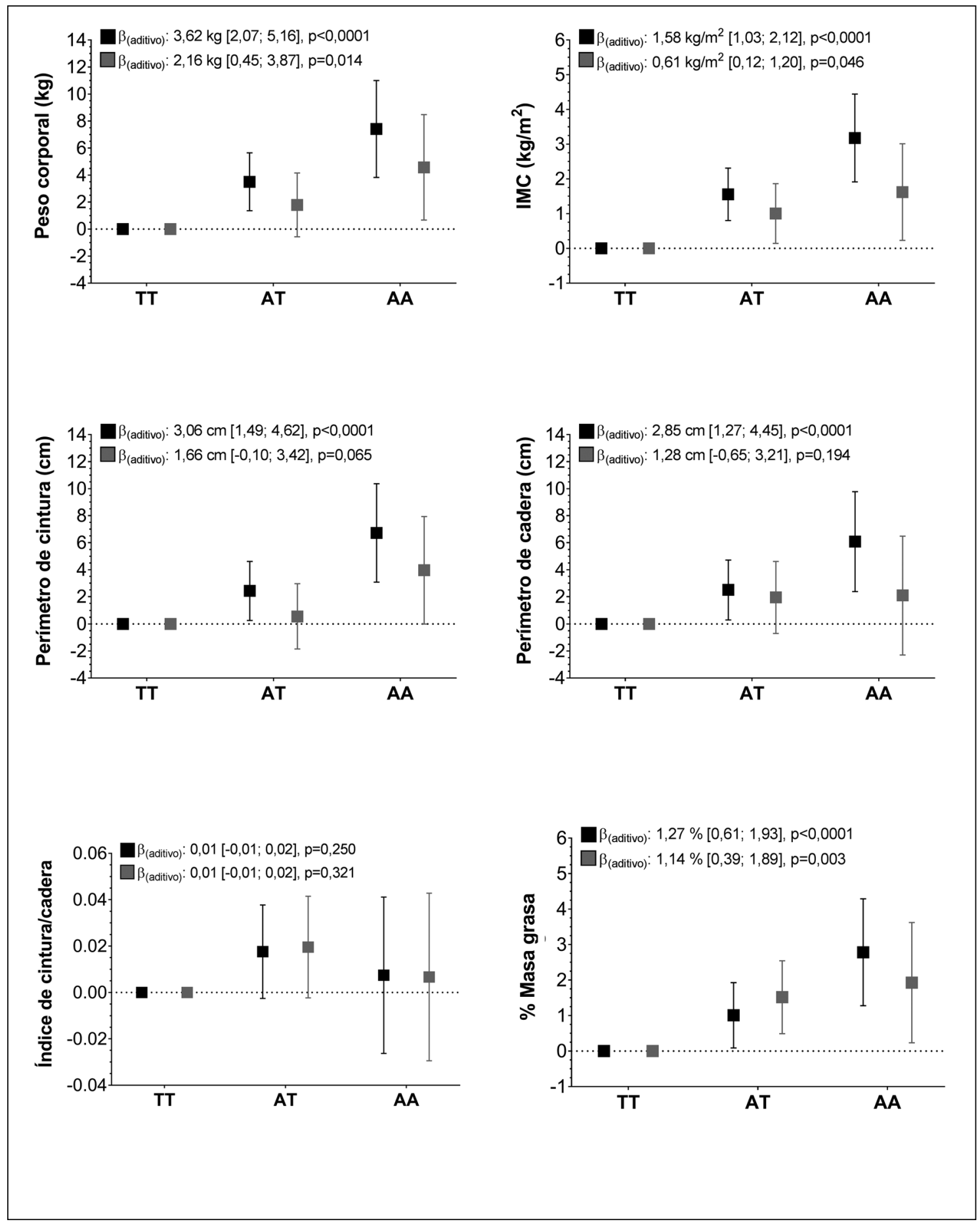

Figura 1. Asociación entre el gen FTO (rs9939609) y marcadores de adiposidad. Los datos son presentados siguiendo un modelo genético aditivo el cual indica el promedio de incremento en la variable de adiposidad por cada copia adicional de la variante de riesgo (A). Los análisis fueron ajustados por edad, sexo, etnia, nivel educacional, ingreso económico, nivel socioeconómico, zona de residencia (urbano/rural), actividad física, tiempo sedente, tabaquismo, consumo de energía, proteínas, grasas totales, carbohidratos complejos, carbohidratos simples y alcohol (Modelo 3). 
Tabla 3. Asociación entre el gen FTO (rs9939609) y marcadores de adiposidad estandarizados a z-score

\begin{tabular}{|c|c|c|c|c|c|}
\hline \multirow[b]{2}{*}{ Variables } & \multicolumn{3}{|c|}{ Genotipo del FTO (rs9939609) } & \multirow{2}{*}{$\begin{array}{l}\text { Efecto del modelo } \\
\text { genético aditivo }\end{array}$} & \multirow[t]{2}{*}{ Valor $\mathbf{p}$} \\
\hline & TT & AT & $\mathbf{A A}$ & & \\
\hline \multicolumn{6}{|c|}{ Peso corporal (SD) } \\
\hline Modelo 0 & 1,00 (Ref.) & $0,34(0,13 ; 0,54)$ & $0,71(0,37 ; 1,06)$ & $0,35(0,20 ; 0,50)$ & $<0,0001$ \\
\hline Modelo 1 & 1,00 (Ref.) & $0,24(0,03 ; 0,45)$ & $0,71(0,34 ; 1,08)$ & $0,31(0,15 ; 0,47)$ & $<0,0001$ \\
\hline Modelo 2 & 1,00 (Ref.) & $0,12(-0,11 ; 0,34)$ & $0,42(0,06 ; 0,77)$ & $0,19(0,03 ; 0,34)$ & 0,022 \\
\hline Modelo 3 & 1,00 (Ref.) & $0,17(-0,06 ; 0,40)$ & $0,44(0,06 ; 0,81)$ & $0,21(0,04 ; 0,37)$ & 0,014 \\
\hline \multicolumn{6}{|l|}{ IMC (SD) } \\
\hline Modelo 0 & 1,00 (Ref.) & $0,42(022 ; 0,62)$ & $0,86(0,52 ; 1,20)$ & $0,42(0,27 ; 0,57)$ & $<0,0001$ \\
\hline Modelo 1 & 1,00 (Ref.) & $0,33(0,11 ; 0,54)$ & $0,55(0,18 ; 0,93)$ & $0,30(0,14 ; 0,45)$ & $<0,0001$ \\
\hline Modelo 2 & 1,00 (Ref.) & $0,19(-0,03 ; 0,41)$ & $0,31(-0,04 ; 0,66)$ & $0,16(0,01 ; 0,32)$ & 0,040 \\
\hline Modelo 3 & 1,00 (Ref.) & $0,21(-0,01 ; 0,43)$ & $0,29(-0,07 ; 0,65)$ & $0,16(0,00 ; 0,32)$ & 0,046 \\
\hline \multicolumn{6}{|c|}{ Perímetro de cintura (SD) } \\
\hline Modelo 0 & 1,00 (Ref.) & $0,22(0,24 ; 0,42)$ & $0,61(0,28 ; 0,95)$ & $0,28(0,14 ; 0,42)$ & $<0,0001$ \\
\hline Modelo 1 & 1,00 (Ref.) & $0,24(0,03 ; 0,45)$ & $0,54(0,18 ; 0,91)$ & $0,26(0,10 ; 0,42)$ & 0,001 \\
\hline Modelo 2 & 1,00 (Ref.) & $0,09(-0,14 ; 0,32)$ & $0,41(0,03 ; 0,78)$ & $0,17(0,01 ; 0,34)$ & 0,040 \\
\hline Modelo 3 & 1,00 (Ref.) & $0,05(-0,17 ; 0,27)$ & $0,36(-0,00 ; 0,73)$ & $0,15(-0,01 ; 0,31)$ & 0,065 \\
\hline \multicolumn{6}{|c|}{ Perímetro de cadera (SD) } \\
\hline Modelo 0 & 1,00 (Ref.) & $0,24(0,03 ; 0,44)$ & $0,57(0,22 ; 0,92)$ & $0,27(0,12 ; 0,42)$ & $<0,0001$ \\
\hline Modelo 1 & 1,00 (Ref.) & $0,24(0,02 ; 0,45)$ & $0,42(0,04 ; 0,80)$ & $0,22(0,06 ; 0,38)$ & 0,008 \\
\hline Modelo 2 & 1,00 (Ref.) & $0,20(-0,05 ; 0,45)$ & $0,27(-0,13 ; 0,67)$ & $0,15(-0,02 ; 0,33)$ & 0,091 \\
\hline Modelo 3 & 1,00 (Ref.) & $0,18(-0,07 ; 0,43)$ & $0,20(-0,22 ; 0,61)$ & $0,12(-0,06 ; 0,30)$ & 0,194 \\
\hline \multicolumn{6}{|c|}{ Índice de cintura/cadera (SD) } \\
\hline Modelo 0 & 1,00 (Ref.) & $0,16(-0,02 ; 0,35)$ & $0,07(-0,24 ; 0,38)$ & $0,08(-0,06 ; 0,21)$ & 0,250 \\
\hline Modelo 1 & 1,00 (Ref.) & $0,18(0,00 ; 0,36)$ & $0,11(-0,20 ; 0,42)$ & $0,10(-0,03 ; 0,23)$ & 0,137 \\
\hline Modelo 2 & 1,00 (Ref.) & $0,22(0,02 ; 0,42)$ & $0,11(-0,21 ; 0,43)$ & $0,10(-0,04 ; 0,24)$ & 0,153 \\
\hline Modelo 3 & 1,00 (Ref.) & $0,18(-0,02 ; 0,38)$ & $0,06(-0,27 ; 0,40)$ & $0,07(-0,07 ; 0,22)$ & 0,321 \\
\hline \multicolumn{6}{|c|}{ \% masa grasa (SD) } \\
\hline Modelo 0 & 1,00 (Ref.) & $0,21(0,02 ; 0,41)$ & $0,59(0,27 ; 0,91)$ & $0,27(0,13 ; 0,41)$ & $<0,0001$ \\
\hline Modelo 1 & 1,00 (Ref.) & $0,28(0,09 ; 0,47)$ & $0,62(0,28 ; 0,95)$ & $0,30(0,16 ; 0,44)$ & $<0,0001$ \\
\hline Modelo 2 & 1,00 (Ref.) & $0,31(0,10 ; 0,51)$ & $0,45(0,12 ; 0,77)$ & $0,25(0,10 ; 0,39)$ & 0,001 \\
\hline Modelo 3 & 1,00 (Ref.) & $0,32(0,10 ; 0,54)$ & $0,41(0,05 ; 0,77)$ & $0,24(0,08 ; 0,40)$ & 0,003 \\
\hline
\end{tabular}

Datos presentados como coeficiente beta estandarizado y $95 \%$ IC según genotipo. El modelo genético aditivo indica el promedio de incremento en la variable de adiposidad expresada en desviación estándar por cada copia adicional de la variante de riesgo (A). Este efecto aditivo y su respectivo 95\% IC fue determinado mediante regresión lineal. Los análisis fueron ajustados por: Modelo 0 - sin ajustar; Modelo 1 -ajustado por edad, sexo, etnia, nivel educacional, ingreso económico, nivel socioeconómico, y zona de residencia (urbano/rural); Modelo 2 - ajustado por el Modelo 1 pero también por actividad física, tiempo sedente y tabaquismo; Modelo 3 - fue ajustado por el Modelo 2 pero también por consumo de energía, proteínas, grasas totales, carbohidratos complejos, carbohidratos simples y alcohol.

y el índice de cintura/cadera para ninguno de los modelos estadísticos (Tabla 2).

Para determinar qué marcador de adiposidad presentaba una mayor asociación con el gen FTO, estos fueron transformados a $z$-score. Tras ajustar los modelos por todas las variables de confusión
(Modelo 3) la mayor asociación con el gen FTO se observó para porcentaje de masa grasa $(0,24$ unidades de DE), seguida por peso corporal $(0,21$ unidades de DE) e IMC (0,16 unidades de DE) por cada copia extra del alelo de riesgo (Tabla 3 y Figura 2). 


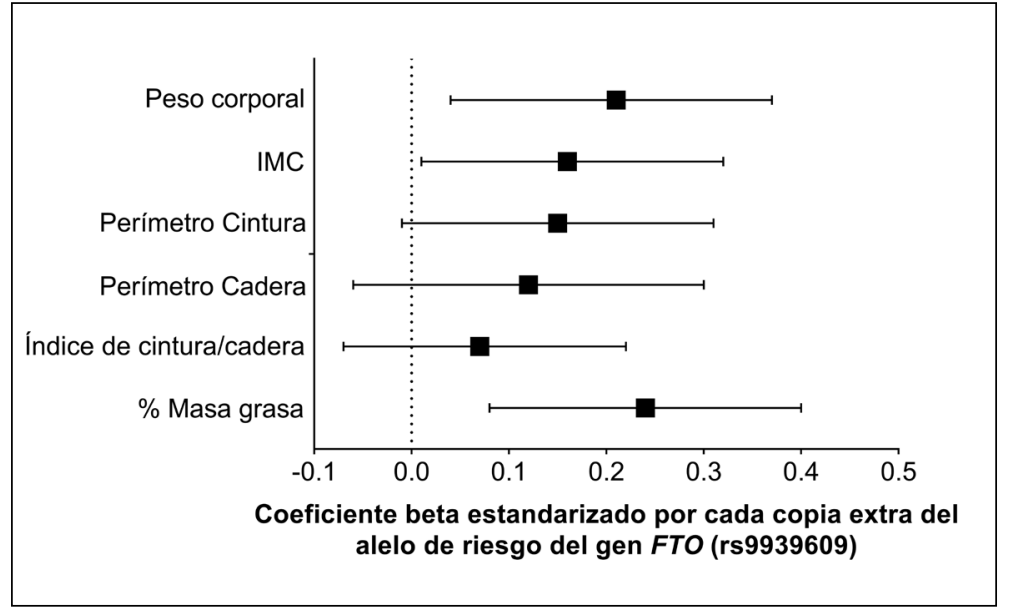

Figura 2. Asociación entre el gen FTO (rs9939609) y marcadores de adiposidad estandarizados a $z$-score. Los análisis fueron ajustados por edad, sexo, etnia, nivel educacional, ingreso económico, nivel socioeconómico, zona de residencia (urbano/rural), actividad física, tiempo sedente, tabaquismo, consumo de energía, proteínas, grasas totales, carbohidratos complejos, carbohidratos simples y alcohol (Modelo 3).

\section{Discusión}

Los principales hallazgos de este estudio confirman que la variante rs9939609 del gen FTO se asocia significativamente con un incremento en el peso corporal, IMC y porcentaje de masa grasa en población adulta chilena, independiente de factores de confusión. Tal asociación no se identificó para el índice de cintura/cadera y disminuyó para PC y perímetro de cadera luego de ajustar los modelos estadísticos por las distintas variables de confusión. Considerando que 74,2\% de la población chilena presenta actualmente algún grado de sobrepeso u obesidad ${ }^{4}$, estos resultados proponen la necesidad de implementar un protocolo de genotipificación que permita la detección temprana de individuos con una mayor predisposición genética a desarrollar obesidad, de forma que intervenciones personalizadas sean llevadas a cabo desde primeras etapas en estos individuos para retrasar o disminuir el desarrollo de obesidad.

Los resultados de este estudio están en concordancia con otras investigaciones que muestran asociación del gen FTO con marcadores de adiposidad en población europea, norteamericana y latina ${ }^{24,25}$. Frayling et al. ${ }^{13}$ determinaron en población europea que los homocigotos para el alelo de riesgo del SNP rs9939609 pesaban $3 \mathrm{~kg}$ de peso adicional, valor que fue menor a lo observado para el Modelo 3 (totalmente ajustado) en este estudio de población chilena, es decir $2,16 \mathrm{~kg}$ para cada copia de alelo A (4,32 kg genotipo AA-Tabla 2).
La asociación del gen FTO y el desarrollo de obesidad fue inicialmente descrita en una población europea y posteriormente confirmada en otras poblaciones de ascendencia no europea ${ }^{13,26,27}$. No obstante, estudios posteriores realizados en poblaciones asiáticas y africanas han mostrado resultados contradictorios respecto a la asociación entre el gen $F T O$ y obesidad ${ }^{16,28}$, resaltando la necesidad de analizar la asociación existente entre este gen con marcadores de adiposidad en distintas poblaciones. En Chile, un estudio previo realizado por Riffo et al., en 374 niños, muestra una asociación positiva entre el gen $F T O$ con el puntaje Z del IMC, en población obesa, pero no en población infantil con peso normal. Resultados similares fueron observados para porcentaje de masa grasa ${ }^{29}$. Sin embargo, hasta la fecha no se han reportado otros estudios en población adulta en Chile, siendo este el primer estudio en confirmar esta asociación entre el gen FTO y marcadores de adiposidad en población adulta.

La alta prevalencia de variantes de riesgo del gen FTO en población adulta en Chile podría ser una oportunidad para la implementación de intervenciones personalizadas basadas en el genotipo FTO de la población, como ha sido demostrado por el estudio Food4Me. Este estudio reportó que personas que reciben recomendaciones personalizadas basadas en su genotipo logran una mayor reducción de su peso corporal en comparación a aquellas que recibieron una recomendación no personalizada ${ }^{30}$. Este estudio también señaló que, en comparación a portadores de la variante protec- 
tora del gen $F T O$, aquellos que presentaron la variable de riesgo (AA) lograron una mayor pérdida de peso independiente del tipo de recomendación que recibieran ${ }^{30}$. A pesar de este alentador avance por parte del grupo Food $4 M e$, actualmente hay escasa evidencia sobre el tipo de dieta específica que podría optimizar la reducción del peso corporal en portadores de alelos de susceptibilidad ${ }^{23,31}$, siendo aún necesario investigar nuevas estrategias personalizadas para esta población.

Entre las limitaciones de este trabajo se encuentra el tipo de estudio realizado y el reducido tamaño de la muestra, lo que pudo haber limitado el poder estadístico de algunos resultados, como es el caso de la asociación entre el gen FTO y el perímetro de cintura. Además, el limitado tamaño de la muestra no permitió realizar un análisis comparativo entre el polimorfismo y marcadores de adiposidad en población mapuche y europea. No obstante, la muestra con la que se trabajó fue suficiente para demostrar una asociación entre el gen FTO con otros marcadores de adiposidad. Otra limitación de este estudio fue solo la evaluación del polimorfismo rs9939609 localizado en el primer intrón del gen $F T O$, a pesar de que el gen FTO es altamente polimórfico en toda su extensión, especialmente en los intrones 1 y $8^{32}$.

Finalmente, el presente estudio presenta la primera evidencia entre la asociación del gen FTO y marcadores de adiposidad en población adulta chilena independiente de factores de confusión. Considerando la alta prevalencia de obesidad existente en Chile y a nivel mundial, próximos estudios deberán investigar estrategias personalizadas para la reducción del peso corporal y otros marcadores de adiposidad en esta población.

\section{Referencias}

1. The Lancet Diabetes E. Should we officially recognise obesity as a disease? Lancet Diabetes Endocrinol 2017; 5 (7): 483.

2. WHO. Obesity: preventing and managing the global epidemic. Report of a WHO consultation. 2000. Report $\mathrm{N}^{\circ}:$ 0512-3054.

3. OECD. Obesity Update 2017. Organization for Economic Co-operation and Development. 2017.

4. MINSAL. Encuesta Nacional de Salud 2016-2017. Ministerio de Salud, Chile. 2017.

5. OMS/OPS. Panorama de la Seguridad Alimentaria y
Nutricional en América Latina y el Caribe. Santiago, Chile: Organización de las Naciones Unidas para la Alimentación y la Agricultura, Organización Panamericana de la Salud; 2016 Enero 2017. Report No.: 1.

6. Alberti KG, Eckel RH, Grundy SM, Zimmet PZ, Cleeman JI, Donato KA, et al. Harmonizing the metabolic syndrome: a joint interim statement of the International Diabetes Federation Task Force on Epidemiology and Prevention; National Heart, Lung, and Blood Institute; American Heart Association; World Heart Federation; International Atherosclerosis Society; and International Association for the Study of Obesity. Circulation. 2009; 120 (16): 1640-5.

7. Dhalwani NN, Zaccardi F, O'Donovan G, Carter P, Hamer M, Yates T, et al. Association Between Lifestyle Factors and the Incidence of Multimorbidity in an Older English Population. J Gerontol A Biol Sci Med Sci 2017; 72 (4): 528-34.

8. Petemann F, Durán E, Labraña AM, Martínez MA, Leiva AM, Garrido-Méndez A, et al. Factores asociados al desarrollo de obesidad en Chile: resultados de la Encuesta Nacional de Salud 2009-2010. Rev Med Chile 2017; 145 (6).

9. Cambron A. [The Human Genome Project and the right to intellectual property]. Rev Derecho Genoma Hum 2000; 13: 79-102.

10. Yeo GS. The role of the FTO (Fat Mass and Obesity Related) locus in regulating body size and composition. Mol Cell Endocrinol 2014; 397 (1-2): 34-41.

11. Hotta K, Nakata Y, Matsuo T, Kamohara S, Kotani K, Komatsu R, et al. Variations in the FTO gene are associated with severe obesity in the Japanese. J Hum Genet 2008; 53 (6): 546-53.

12. Jonsson $\mathrm{A}$, Renstrom $\mathrm{F}$, Lyssenko $\mathrm{V}$, Brito $\mathrm{EC}$, Isomaa $\mathrm{B}$, Berglund G, et al. Assessing the effect of interaction between an FTO variant (rs9939609) and physical activity on obesity in 15,925 Swedish and 2,511 Finnish adults. Diabetologia 2009; 52 (7): 1334-8.

13. Frayling TM, Timpson NJ, Weedon MN, Zeggini E, Freathy RM, Lindgren CM, et al. A common variant in the FTO gene is associated with body mass index and predisposes to childhood and adult obesity. Science 2007; 316 (5826): 889-94.

14. González-Sánchez JL, Zabena C, Martínez-Larrad MT, Martínez-Calatrava MJ, Pérez-Barba M, Serrano-Ríos M. Variant rs9939609 in the FTO gene is associated with obesity in an adult population from Spain. Clinical Endocrinology 2009; 70 (3): 390-3.

15. Liu AL, Xie HJ, Xie HY, Liu J, Yin J, Hu JS, et al. Association between fat mass and obesity associated (FTO) gene rs9939609 A/T polymorphism and polycystic ovary 
syndrome: a systematic review and meta-analysis. BMC Medical Genetics 2017; 18: 89.

16. Yang $\mathrm{M}, \mathrm{Xu} \mathrm{Y}$, Liang L, Fu J, Xiong F, Liu G, et al. The Effects of Genetic Variation in FTO rs9939609 on Obesity and Dietary Preferences in Chinese Han Children and Adolescents. PloS one 2014; 9 (8).

17. Celis-Morales CA, Perez-Bravo F, Ibanes L, Sanzana R, Hormazabal E, Ulloa N, et al. Insulin resistance in Chileans of European and indigenous descent: evidence for an ethnicity x environment interaction. PloS one 2011; 6 (9): e24690.

18. Marfell-Jones MJ, Stewart A, De Ridder J. International standards for anthropometric assessment. The International Society for the Advancement of Kinanthropometry. Australia. 2012.

19. WHO. Obesity: preventing and managing the global epidemic. World Health Organization. 2000; Disponible en: http://www.who.int/nutrition/publications/obesity/ WHO_TRS_894/en/.

20. Durnin JV, Womersley J. Body fat assessed from total body density and its estimation from skinfold thickness: measurements on 481 men and women aged from 16 to 72 years. Br J Nutr 1974; 32 (1): 77-97.

21. Buckley J, Sim J, Eston R, Hession R, Fox R. Reliability and validity of measures taken during the Chester step test to predict aerobic power and to prescribe aerobic exercise. Br J Sport Med 2004; 38 (2): 197-205.

22. Freedson PS, Melanson E, Sirard J. Calibration of the Computer Science and Applications, Inc. accelerometer. Med Sci Sports Exerc 1998; 30 (5): 777-81.

23. Przeliorz-Pyszczek A, Regulska-Ilow B. The role of macronutrient intake in reducing the risk of obesity and overweight among carriers of different polymorphisms of FTO gene. A review. Rocz Panstw Zakl Hig 2017; 68 (1): 5-13.

24. Vilella M, Nunes de Oliveira Costa G, Lima Barreto M, Alexandrina Figueredo C, Maria Alcantara-Neves N, Cunha Rodrigues L, et al. Effect of dietary consumption as a modifier on the association between FTO gene variants and excess body weight in children from an admixed population in Brazil: the Social Changes, Asthma and Allergy in Latin America (SCAALA) cohort study. Br J Nutr 2017; 117 (11): 1503-10.

25. Mejía-Benítez A, Klünder-Klünder M, Yengo L, Meyre D, Aradillas C, Cruz E, et al. Analysis of the contribution of FTO, NPC1, ENPP1, NEGR1, GNPDA2 and MC4R genes to obesity in Mexican children. BMC Med Genet 2013; 14: 21 .

26. Dina C, Meyre D, Gallina S, Durand E, Körner A, Jacobson $\mathrm{P}$, et al. Variation in FTO contributes to childhood obesity and severe adult obesity. Nat Genet 2007; 39 (6): 724-6.

27. Scuteri A, Sanna S, Chen WM, Uda M, Albai G, Strait $\mathrm{J}$, et al. Genome-wide association scan shows genetic variants in the FTO gene are associated with obesity-related traits. PLoS Genet 2007; 3 (7): e115.

28. Li H, Wu Y, Loos RJ, Hu FB, Liu Y, Wang J, et al. Variants in the fat mass- and obesity-associated (FTO) gene are not associated with obesity in a Chinese Han population. Diabetes 2008; 57 (1): 264-8.

29. Riffo B, Asenjo S, Sáez K, Aguayo C, Muñoz I, Bustos $\mathrm{P}$, et al. FTO gene is related to obesity in Chilean Amerindian children and impairs HOMA-IR in prepubertal girls. Pediatr Diabetes 2012; 13 (5): 384-91.

30. Celis-Morales C, Marsaux CF, Livingstone KM, Navas-Carretero S, San-Cristobal R, Fallaize R, et al. Can genetic-based advice help you lose weight? Findings from the Food4Me European randomized controlled trial. Am J Clin Nutr 2017; 105 (5): 1204-13.

31. Livingstone KM, Celis-Morales C, Papandonatos GD, Erar B, Florez JC, Jablonski KA, et al. FTO genotype and weight loss: systematic review and meta-analysis of 9563 individual participant data from eight randomised controlled trials. BMJ 2016; 354: i4707.

32. Adeyemo A, Chen G, Zhou J, Shriner D, Doumatey A, Huang $\mathrm{H}$, et al. FTO genetic variation and association with obesity in West Africans and African Americans. Diabetes 2010; 59 (6): 1549-54. 